\title{
Argon Isotopic Dating of Neolithic Jade Artifacts and Raw Materials from Eastern China and Its Implications
}

\author{
Su-Jung Chou ${ }^{1}$, Ching-Hua Lo ${ }^{1,{ }^{*}}$, Yun-Ao He ${ }^{2}$, Jing-Guo Zhang ${ }^{3}$, Yoshiyuki Iizuka ${ }^{4}$, \\ $\mathrm{Ju}-\mathrm{Yu} \mathrm{Na}{ }^{2}$, and Tsung-Kuang $\mathrm{Yeh}^{5}$ \\ ${ }^{1}$ Institute of Geosciences, National Taiwan University, Taipei, Taiwan, ROC \\ ${ }^{2}$ Department of History, Nanjing University, Nanjing, China \\ ${ }^{3}$ Institute of Archeology, Hefei, China \\ ${ }^{4}$ Institute of Earth Sciences, Academia Sinica, Taipei, Taiwan, ROC \\ ${ }^{5}$ Nuclear Science and Technology Development Center, National Tsing Hua University, Hsinchu, Taiwan, ROC
}

Received 25 April 2008, accepted 7 July 2008

\begin{abstract}
The origin of Chinese culture during the Neolithic age has long been a focus of academic debate. The controversy is centered on whether the origins of Neolithic culture in China were singular or more diverse. Consequently, understanding the spatial distribution of archaeological jade artifacts in the context of the sources of raw jade has been one of the most reasonable means to infer the routes of cultural migration and trade activities during the Neolithic period. It was widely advocated that HeTian Jade from XinJiang was the sole source of jade for all of Neolithic China. However, the discovery of jade mines at MeiLing (JiangSu Province) in 1989 raises the question as to whether raw jade could have come from an alternate source other than XinJiang during this period. Using ${ }^{40} \mathrm{Ar}{ }^{39} \mathrm{Ar}$ laser dating technique, this study attempts to correlate the formation ages of excavated jade artifacts at LingJiaTan (AnHui Province) to the nearby MeiLing jade mines and faraway HeTian jade mines, in the hope of tracing the possible culture interactions and trading activities among these areas. The results show that the formation ages of LingJiaTan jade artifacts are around $120 \mathrm{Ma}$, about the same age as jade from the nearby MeiLing jade mines. In addition, both jades are tremolite with similar chemical compositions and mineral characteristics. Therefore, the material source of jade artifacts excavated at LingJiaTan archeological site most likely originated from these nearby MeiLing jade mines. By contrast, there is a big difference in the formation ages between Meiling jade artifacts and raw HeTian Jade (277.3 Ma). Based on these results, we conclude that in the Neolithic age, people living in LingJiaTan (AnHui Province) probably had interactions with people around MeiLing (JiangSu Province), but not with peoples of XinJiang Province.
\end{abstract}

Key words: Ar-Ar dating, Archaic jades, China

Citation: Chou, S. J., C. H. Lo, Y. A. He, J. G. Zhang, Y. Iizuka, J. Y. Na, and T. K. Yeh, 2009: Argon isotopic dating of neolithic jade artifacts and raw materials from eastern China and its implications. Terr. Atmos. Ocean. Sci., 20, 501-509, doi: 10.3319/TAO.2008.07.07.01(TT)

\section{INTRODUCTION}

In recent decades, large quantities of jade artifacts have been unearthed at more than two dozen Neolithic archaeological sites in China. An outstanding issue concerning archaeologists is how to trace the raw materials used to make these artifacts. Previous studies often assumed that HeTian of XinJiang Province was the sole source of raw jade for the whole Neolithic China (Na 1980; Yang 1998). If this assumption were true, artisans living in the vicinity of the aforementioned archeological sites must have sourced their

\footnotetext{
* Corresponding author

E-mail:loch@ntu.edu.tw
}

jade either directly or indirectly from XinJiang Province. This means that XinJiang Province would have been at the center of Neolithic culture in China. On the other hand, if raw jade material were sourced from local mines, this would indicate more diverse origins for the development of Neolithic culture in China (Fig. 1). Recent studies have found many jade mines around China (Kolesnik 1966). A total of 43 mines in XinJiang Province have been discovered and the jades sourced from these mines have been called HeTian Jade. In 1989, ShiaoMeiLing Village of LiYang City, JiangSu Province, was also recognized as being one of the sites among many Neolithic jade culture clusters. 


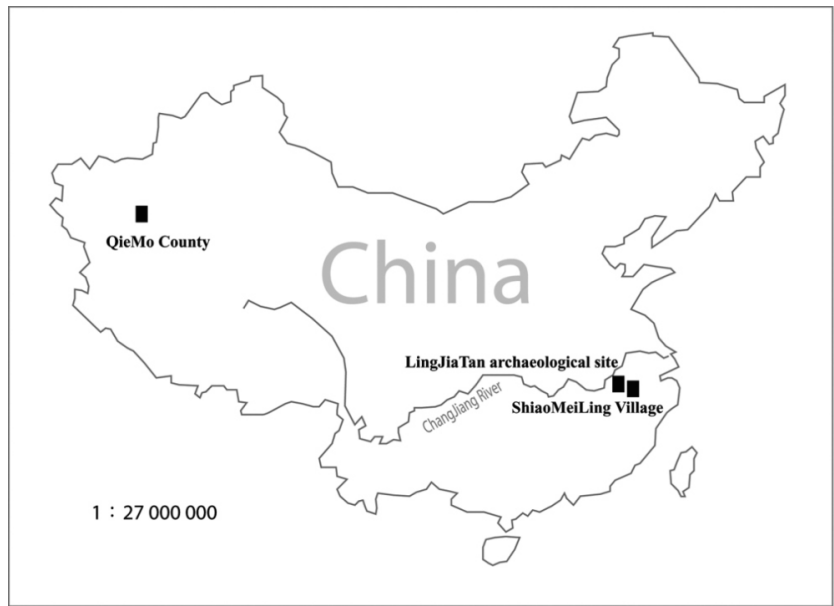

Fig. 1. Geographical distribution of LingJiaTan archaeological site and jade mines in China.

The jade sourced from the mines found in the region is called MeiLing Jade (Cui et al. 2002; Zhong and Zhang 2002). As a result of the new discovery of two possible raw jade mines, an important and interesting archeological research topic has been raised: delineating the raw jade sources from at least the two known sites (Fig. 1).

In the present study, archaic jade artifacts were unearthed from LingJiaTan archaeological site in the AnHui Province, an important ancient cultural center (AnHui Provincial Institute of Cultural Relics 2000). ${ }^{40} \mathrm{Ar} /{ }^{39} \mathrm{Ar}$ laser dating analysis was used to date these archaic jade samples and raw jade materials of various jade mines. By comparing these analysis results with current geological data, the source of raw jade materials used in the archaic jade artifacts can be identified. Hopefully, the study can offer a better understanding of the cultural migration and economic activities of these Neolithic people.

\section{ARCHEOLOGICAL JADE ARTIFACTS AND OCCURRENCES OF RAW JADE MATERIALS}

\subsection{LingJiaTan Archaeological Site}

The LingJiaTan archaeological site is located at ChihRen Village, TongJa Town, HanSan County, AnHui Province. It was a Neolithic settlement around 5200 to 5500 years ago (AnHui Provincial Institute of Cultural Relics 2000). From 1985 to 2000, AnHui Provincial Institute of Cultural Relics carried out 4 archaeological digs at the site. About 1500 pieces of jade and ceramic artifices were unearthed (Anhui Provincial Institute of Cultural Relics 2000). During the excavations, all the jade artifacts excavated from AnHui LingJiaTan archaeological site were carefully examined on site. As expected, the prolonged burial of the artifacts has induced weathering to produce secondary minerals. Some of the jade artifacts exhibit properties which are not uniform, including layered structures and chicken-white coloring.

\subsection{MeiLing Jades}

The MeiLing Jade lode is exposed at ShiaoMeiLing Village, located in the Southeast region of PinChiao County, LiYang City, JiangSu Province. The mines are located in the TienMo mountain range, south of NanKing City, and southwest of LiYang City, JiangSu Province. The discovery of MeiLing Jade occurred whilst mining iron ore in the last century. In the outcrop, the host rocks are mainly composed of shallow marine limestone and dolomite beds of the Permian Qixia Formation. After deposition, these carbonate beds were intruded by Miaoshi granite during the Yanshanian orogeny (Zhong 2000; Cui et al. 2002; Zhong and Zhang 2002). Through chemical reactions of the carbonate beds with molten fluids during contact metamorphism, the MeiLing Jade lodes and iron skarns were formed between the limestone beds and the granite pluton. The skarn layers along with the jade lodes were highly disturbed by faults. The jade lode is wedge shaped being approximately 50 $90 \mathrm{~cm}$ in width. Field evidence suggests that the jade lode is a part of the skarns, resultant from contact metamorphism between Qixia Limestone and Yanshanian granite emplacement (Zhong 2000; Cui et al. 2002; Zhong and Zhang 2002).

\subsection{QieMo (XinJiang) Jades}

XinJiang in China is an area well known for its production of excellent raw jade materials. HeTian Jade is the general name for the tremolite jade materials produced at KunLun Mountains and ArJin Mountains. The known outcrops of jade beds are mainly located in five areas, three at KunLun Mountains (ShaCheh-YehChen, PeiSan-HeTian, and CheiLo-YuTian) and two at ArJin Mountains (QieMo, RoJiang) (Tang et al. 2002). The HeTian jade used in the present study was collected from the QieMo area and is named after the area.

Previous studies reported that HeTian jade lodes are also produced at the contact zones between late Paleozoic acidic igneous rocks (granite, granitic diorite and diorite) and magnesium carbonates (Tang et al. 2002). Magnesium carbonate rocks are mainly Proterozoic dolomite marbles. The tremolite jade was generated by metamorphic reactions between dolomite marbles and acidic molten magma. It is reported that the skarns consist of several reactions zones, from an inner feldspar-rich layer close to the granitic body, through the layers of diopside, tremolite, serpentine rich zones, toward the dolomitic marble layer near the carbonate host rocks and that the jade lode is found in the tremolite zone (Tang et al. 2002).

\section{SAMPLES}

Three raw jade material samples from jade mines (MLG1 and MLW1 from ShiaoMeiLing and CM1 from HeTian) were acquired for the present study. Two of them (MLG1, 
MLW1) were collected from jade mines near ShiaoMeiLing Village of LiYang County, JiangSu Province. The first sample (MLG1) shows light green in color. The second sample (MLW1) exhibits grayish white color. Whereas, the third sample, a piece of HeTian tremolite jade (CM1) with light green coloring was taken from a jade mine in the mountain area of QieMo County in XinJiang Province. To examine their petrographic features and mineral compositions, thin sections were prepared, and then carefully examined under a light microscope and secondary electron microscope, and further analyzed chemically using an electron microprobe. All the samples appear to be massive in texture, with similar mineral composition that mainly consists of tremolite (Fig. 2).

The archaic jade artifacts used for sourcing purposes,

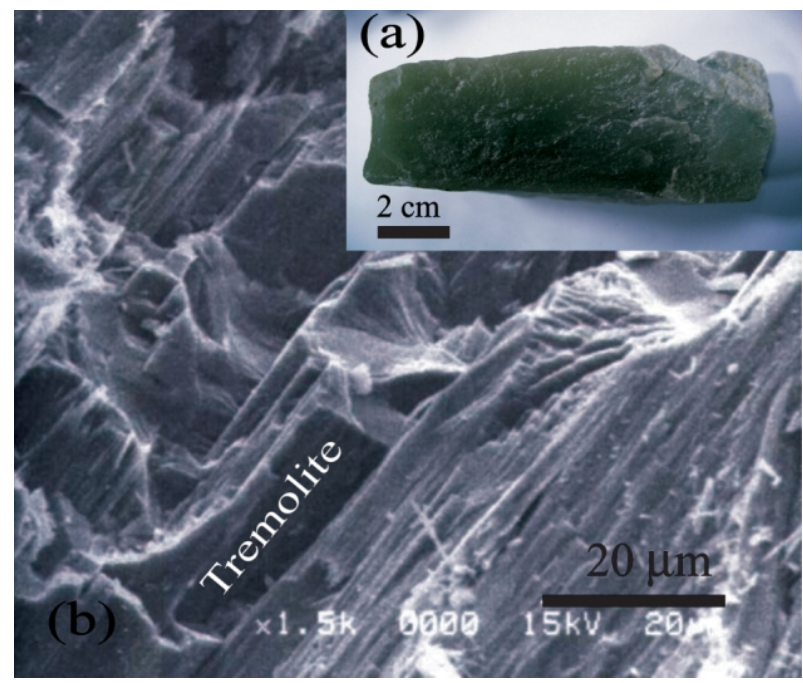

Fig. 2. (a) Photograph of hand specimen for the raw jade sample MLG1 collected from ShiaoMeiLing Village of LiYang County, JiangSu Province. (b) SEM image showing massive texture of tremolite in the sample MLG1. two broken pieces of ring-shaped archaic artifact (LJT2 and LJT35) from LingJiaTan archaeological site, were granted courtesy of the AnHui Provincial Institute of Cultural Relics. Both samples display pale-green coloring with visible white-color and pale-brown alternating layers (Fig. 3a). Nevertheless, they still exhibit similar massive textures and mineral compositions, which are similar to those collected from the outcrops, except the archaic jade samples appear to be softening with many visible micro-pores filled with alteration products (Figs. 3b, c). X-ray diffraction experiments show that the alteration products are mainly chlorite, kaolinite, calcite and some unidentified amorphous phases, which are findings similar to those reported by Fong et al. (2005).

\section{ANALYTICAL METHODS}

In order to obtain the formation ages of the jade samples, laser ${ }^{40} \mathrm{Ar} /{ }^{39} \mathrm{Ar}$ single-grain dating technique is adopted in the present study. Tremolite separates were first extracted from samples by hand-picking and then irradiated along with LP-6 biotite standards with ${ }^{40} \mathrm{Ar} /{ }^{39} \mathrm{Ar}$ age of $128.4 \pm 0.2 \mathrm{Ma}$ (Renne et al. 1998), in the VT-C position for 30 hours at the THOR reactor, Hsing-Hua University. After irradiation, standards and samples were totally fused grain-by-grain using a US LASER Nd-YAG laser operated in continuous mode. The gas was analyzed on a VG 3600 mass spectrometer at the National Taiwan University. Detailed analytical procedures are outlined by Lo et al. (2002). The results of the ${ }^{40} \mathrm{Ar} /{ }^{39} \mathrm{Ar}$ laser singlegrain fusion experiments are given in Table 1, and plotted as isotope correlation diagrams in Figs 4 and 5. All the analytical results are summarized in Table 2.

\section{RESULTS AND DISCUSSIONS}

As shown in Fig. 4, all the raw jade materials from out-
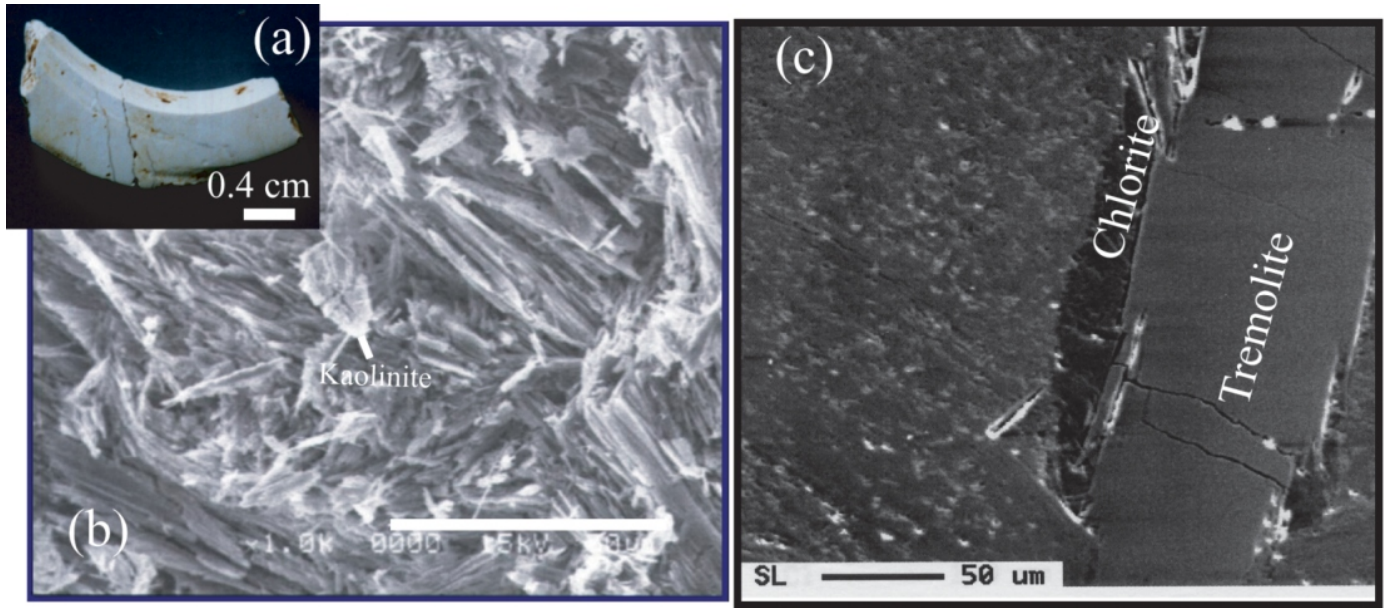

Fig. 3. (a) Photograph of LJT2 archaic jades; (b) SEM; and (c) back scattering image of sample LJT2, showing porous texture of tremolite and its alteration products (kaolinite and chlorite). 
Table 1. Analytical results of laser fusion experiments.

\begin{tabular}{|c|c|c|c|c|c|c|c|}
\hline No. & Atm. \% & ${ }^{36} \mathrm{Ar} /{ }^{39} \mathrm{Ar}$ & ${ }^{37} \mathrm{Ar} /{ }^{39} \mathrm{Ar}$ & ${ }^{38} \mathrm{Ar} /{ }^{39} \mathrm{Ar}$ & ${ }^{40} \mathrm{Ar} /{ }^{39} \mathrm{Ar}$ & ${ }^{40} \mathrm{Ar} /{ }^{36} \mathrm{Ar}$ & Date (Ma) \\
\hline \multicolumn{8}{|c|}{ MLG1 Tremolite } \\
\hline 1 & 35.3 & $5.57 \mathrm{E}-02$ & $2.10 \mathrm{E}+01$ & 2.32E-01 & $4.22 \mathrm{E}+01$ & $7.57 \mathrm{E}+02$ & $117.5 \pm 2.2$ \\
\hline 2 & 38.99 & $5.50 \mathrm{E}-02$ & $2.13 \mathrm{E}+01$ & $2.34 \mathrm{E}-01$ & $3.76 \mathrm{E}+01$ & $6.84 \mathrm{E}+02$ & $99.4 \pm 5.4$ \\
\hline 3 & 30.48 & 4.03E-02 & $1.43 \mathrm{E}+01$ & $1.03 \mathrm{E}-01$ & $3.56 \mathrm{E}+01$ & $8.82 \mathrm{E}+02$ & $106.3 \pm 2.1$ \\
\hline 4 & 19.41 & $2.46 \mathrm{E}-02$ & $1.53 \mathrm{E}+01$ & $9.69 \mathrm{E}-02$ & $3.15 \mathrm{E}+01$ & $1.28 \mathrm{E}+03$ & $109.2 \pm 5.7$ \\
\hline 5 & 35.87 & $5.29 \mathrm{E}-02$ & $2.16 \mathrm{E}+01$ & $2.14 \mathrm{E}-01$ & $3.90 \mathrm{E}+01$ & $7.39 \mathrm{E}+02$ & $108.2 \pm 1.6$ \\
\hline 6 & 40.51 & $6.10 \mathrm{E}-02$ & $1.83 \mathrm{E}+01$ & $1.80 \mathrm{E}-01$ & $4.12 \mathrm{E}+01$ & $6.74 \mathrm{E}+02$ & $105.6 \pm 1.6$ \\
\hline 7 & 10.35 & $1.55 \mathrm{E}-02$ & $1.61 \mathrm{E}+01$ & $1.13 \mathrm{E}-01$ & $3.25 \mathrm{E}+01$ & $2.10 \mathrm{E}+03$ & $124.6 \pm 2.0$ \\
\hline 8 & 32.46 & 4.99E-02 & $2.11 \mathrm{E}+01$ & $2.14 \mathrm{E}-01$ & $4.06 \mathrm{E}+01$ & $8.13 \mathrm{E}+02$ & $118.0 \pm 3.2$ \\
\hline 9 & 15.64 & $2.36 \mathrm{E}-02$ & $2.13 \mathrm{E}+01$ & $1.87 \mathrm{E}-01$ & $3.43 \mathrm{E}+01$ & $1.46 \mathrm{E}+03$ & $124.4 \pm 2.9$ \\
\hline 10 & 30.45 & 4.44E-02 & $2.21 \mathrm{E}+01$ & $2.28 \mathrm{E}-01$ & $3.77 \mathrm{E}+01$ & $8.48 \mathrm{E}+02$ & $113.1 \pm 1.6$ \\
\hline 11 & 25.34 & $3.56 \mathrm{E}-02$ & $2.37 \mathrm{E}+01$ & $2.23 \mathrm{E}-01$ & $3.45 \mathrm{E}+01$ & $9.69 \mathrm{E}+02$ & $111.2 \pm 3.1$ \\
\hline 12 & 26.32 & $3.42 \mathrm{E}-02$ & $2.06 \mathrm{E}+01$ & $1.81 \mathrm{E}-01$ & $3.25 \mathrm{E}+01$ & $9.51 \mathrm{E}+02$ & $103.5 \pm 1.7$ \\
\hline 13 & 41.79 & $6.32 \mathrm{E}-02$ & $2.14 \mathrm{E}+01$ & $2.16 \mathrm{E}-01$ & $4.08 \mathrm{E}+01$ & $6.46 \mathrm{E}+02$ & $102.8 \pm 2.3$ \\
\hline 14 & 16.19 & $2.20 \mathrm{E}-02$ & $1.69 \mathrm{E}+01$ & $1.25 \mathrm{E}-01$ & $3.23 \mathrm{E}+01$ & $1.47 \mathrm{E}+03$ & $116.2 \pm 1.0$ \\
\hline 15 & 41.16 & $6.15 \mathrm{E}-02$ & $2.09 \mathrm{E}+01$ & $2.30 \mathrm{E}-01$ & $4.04 \mathrm{E}+01$ & $6.56 \mathrm{E}+02$ & $102.8 \pm 1.7$ \\
\hline 16 & 26.57 & $3.36 \mathrm{E}-02$ & $1.55 \mathrm{E}+01$ & $1.72 \mathrm{E}-01$ & $3.30 \mathrm{E}+01$ & $9.82 \mathrm{E}+02$ & $104.3 \pm 1.3$ \\
\hline 17 & 31.92 & $4.76 \mathrm{E}-02$ & $2.05 \mathrm{E}+01$ & $2.25 \mathrm{E}-01$ & $3.93 \mathrm{E}+01$ & $8.25 \mathrm{E}+02$ & $115.2 \pm 1.6$ \\
\hline 18 & 24.84 & $3.54 \mathrm{E}-02$ & $2.22 \mathrm{E}+01$ & $2.24 \mathrm{E}-01$ & $3.54 \mathrm{E}+01$ & $1.00 \mathrm{E}+03$ & $114.8 \pm 2.1$ \\
\hline \multicolumn{8}{|c|}{$\mathrm{J}$-value $=0.00243484 \pm 0.00000405$} \\
\hline \multicolumn{8}{|c|}{ Mean Age $=110.9 \mathrm{Ma}(\mathrm{STDEV}=7.5 \mathrm{Ma})$} \\
\hline
\end{tabular}

MLW1 Tremolite

\begin{tabular}{rrrrrrrr}
\hline 1 & 48.31 & $9.56 \mathrm{E}-02$ & $1.28 \mathrm{E}+01$ & $4.94 \mathrm{E}-02$ & $5.65 \mathrm{E}+01$ & $5.91 \mathrm{E}+02$ & $124.4 \pm 1.5$ \\
2 & 28.19 & $3.72 \mathrm{E}-02$ & $8.12 \mathrm{E}+00$ & $3.13 \mathrm{E}-02$ & $3.69 \mathrm{E}+01$ & $9.91 \mathrm{E}+02$ & $112.7 \pm 0.6$ \\
3 & 67.71 & $2.00 \mathrm{E}-01$ & $3.22 \mathrm{E}+00$ & $6.86 \mathrm{E}-02$ & $8.68 \mathrm{E}+01$ & $4.35 \mathrm{E}+02$ & $118.8 \pm 3.0$ \\
4 & 9.27 & $1.19 \mathrm{E}-02$ & $6.32 \mathrm{E}+00$ & $2.78 \mathrm{E}-02$ & $3.29 \mathrm{E}+01$ & $2.76 \mathrm{E}+03$ & $126.5 \pm 0.7$ \\
5 & 12.57 & $1.68 \mathrm{E}-02$ & $9.82 \mathrm{E}+00$ & $2.73 \mathrm{E}-02$ & $3.38 \mathrm{E}+01$ & $2.00 \mathrm{E}+03$ & $125.4 \pm 0.8$ \\
6 & 46.45 & $1.06 \mathrm{E}-01$ & $8.50 \mathrm{E}+01$ & $9.89 \mathrm{E}-02$ & $5.35 \mathrm{E}+01$ & $5.06 \mathrm{E}+02$ & $127.9 \pm 3.5$ \\
7 & 48.5 & $8.88 \mathrm{E}-02$ & $1.34 \mathrm{E}+01$ & $4.56 \mathrm{E}-02$ & $5.20 \mathrm{E}+01$ & $5.86 \mathrm{E}+02$ & $114.5 \pm 1.0$ \\
8 & 67.05 & $1.89 \mathrm{E}-01$ & $3.03 \mathrm{E}+01$ & $7.76 \mathrm{E}-02$ & $7.97 \mathrm{E}+01$ & $4.23 \mathrm{E}+02$ & $113.5 \pm 2.8$ \\
9 & 21.19 & $2.58 \mathrm{E}-02$ & $4.11 \mathrm{E}+00$ & $2.52 \mathrm{E}-02$ & $3.46 \mathrm{E}+01$ & $1.34 \mathrm{E}+03$ & $115.6 \pm 0.4$ \\
10 & 57.06 & $1.28 \mathrm{E}-01$ & $3.93 \mathrm{E}+01$ & $6.70 \mathrm{E}-02$ & $6.13 \mathrm{E}+01$ & $4.78 \mathrm{E}+02$ & $114.5 \pm 2.7$ \\
11 & 29.86 & $4.45 \mathrm{E}-02$ & $1.29 \mathrm{E}+01$ & $3.53 \mathrm{E}-02$ & $4.08 \mathrm{E}+01$ & $9.17 \mathrm{E}+02$ & $122.0 \pm 1.1$ \\
12 & 26.81 & $4.37 \mathrm{E}-02$ & $3.89 \mathrm{E}+01$ & $4.33 \mathrm{E}-02$ & $3.73 \mathrm{E}+01$ & $8.53 \mathrm{E}+02$ & $118.4 \pm 2.4$ \\
13 & 8.05 & $1.04 \mathrm{E}-02$ & $8.85 \mathrm{E}+00$ & $2.17 \mathrm{E}-02$ & $3.00 \mathrm{E}+01$ & $2.88 \mathrm{E}+03$ & $117.4 \pm 0.5$ \\
14 & 43.79 & $8.13 \mathrm{E}-02$ & $5.29 \mathrm{E}+01$ & $5.73 \mathrm{E}-02$ & $4.58 \mathrm{E}+01$ & $5.63 \mathrm{E}+02$ & $112.8 \pm 2.5$ \\
15 & 57.91 & $1.33 \mathrm{E}-01$ & $5.44 \mathrm{E}+01$ & $8.56 \mathrm{E}-02$ & $6.06 \mathrm{E}+01$ & $4.57 \mathrm{E}+02$ & $112.0 \pm 2.1$ \\
\hline
\end{tabular}


Table 1. (Continued)

\begin{tabular}{|c|c|c|c|c|c|c|c|}
\hline No. & Atm. \% & ${ }^{36} \mathrm{Ar} /{ }^{39} \mathrm{Ar}$ & ${ }^{37} \mathrm{Ar} /{ }^{39} \mathrm{Ar}$ & ${ }^{38} \mathrm{Ar} /{ }^{39} \mathrm{Ar}$ & ${ }^{40} \mathrm{Ar} /{ }^{39} \mathrm{Ar}$ & ${ }^{40} \mathrm{Ar} /{ }^{36} \mathrm{Ar}$ & Date (Ma) \\
\hline \multicolumn{8}{|c|}{ MLW1 Tremolite } \\
\hline 16 & 30.47 & $4.62 \mathrm{E}-02$ & $2.72 \mathrm{E}+01$ & $4.02 \mathrm{E}-02$ & $3.81 \mathrm{E}+01$ & $8.25 \mathrm{E}+02$ & $114.2 \pm 2.1$ \\
\hline 17 & 25.53 & $3.53 \mathrm{E}-02$ & $1.74 \mathrm{E}+01$ & $3.46 \mathrm{E}-02$ & $3.58 \mathrm{E}+01$ & $1.01 \mathrm{E}+03$ & $114.1 \pm 0.6$ \\
\hline 18 & 17.51 & $3.39 \mathrm{E}-02$ & $5.66 \mathrm{E}+01$ & $4.73 \mathrm{E}-02$ & $3.30 \mathrm{E}+01$ & $9.71 \mathrm{E}+02$ & $119.3 \pm 1.3$ \\
\hline 19 & 8.89 & $1.36 \mathrm{E}-02$ & $1.62 \mathrm{E}+01$ & $2.70 \mathrm{E}-02$ & $3.16 \mathrm{E}+01$ & $2.32 \mathrm{E}+03$ & $122.9 \pm 0.5$ \\
\hline \multicolumn{8}{|c|}{$\mathrm{J}$-value $=0.00242459 \pm 0.00000405$} \\
\hline \multicolumn{8}{|c|}{ Mean Age $=118.3 \mathrm{Ma}(\mathrm{STDEV}=5.2 \mathrm{Ma})$} \\
\hline \multicolumn{8}{|c|}{ CM1 Tremolite } \\
\hline 1 & 19.29 & $6.92 \mathrm{E}-02$ & $1.21 \mathrm{E}+02$ & $6.39 \mathrm{E}-02$ & $5.88 \mathrm{E}+01$ & $8.49 \mathrm{E}+02$ & $272.1 \pm 6.5$ \\
\hline 2 & 17.56 & $6.94 \mathrm{E}-02$ & $1.37 \mathrm{E}+02$ & $4.47 \mathrm{E}-02$ & $5.81 \mathrm{E}+01$ & $8.37 \mathrm{E}+02$ & $277.6 \pm 8.4$ \\
\hline 3 & 18.43 & 7.17E-02 & $1.28 \mathrm{E}+02$ & $5.62 \mathrm{E}-02$ & $6.26 \mathrm{E}+01$ & $8.73 \mathrm{E}+02$ & $292.8 \pm 7.1$ \\
\hline 4 & 28.2 & $9.33 \mathrm{E}-02$ & $1.26 \mathrm{E}+02$ & $6.13 \mathrm{E}-02$ & $6.41 \mathrm{E}+01$ & $6.87 \mathrm{E}+02$ & $265.7 \pm 6.8$ \\
\hline 5 & 20.27 & $7.51 \mathrm{E}-02$ & $1.39 \mathrm{E}+02$ & $4.95 \mathrm{E}-02$ & $5.80 \mathrm{E}+01$ & $7.72 \mathrm{E}+02$ & $268.8 \pm 7.3$ \\
\hline 6 & 19.9 & $7.63 \mathrm{E}-02$ & $1.44 \mathrm{E}+02$ & $6.96 \mathrm{E}-02$ & $5.87 \mathrm{E}+01$ & $7.70 \mathrm{E}+02$ & $274.3 \pm 7.5$ \\
\hline 7 & 22.71 & $8.52 \mathrm{E}-02$ & $1.43 \mathrm{E}+02$ & $5.56 \mathrm{E}-02$ & $6.33 \mathrm{E}+01$ & $7.43 \mathrm{E}+02$ & $284.4 \pm 7.3$ \\
\hline 8 & 32.78 & $9.97 \mathrm{E}-02$ & $1.17 \mathrm{E}+02$ & $6.61 \mathrm{E}-02$ & $6.29 \mathrm{E}+01$ & $6.31 \mathrm{E}+02$ & $243.9 \pm 6.7$ \\
\hline 9 & 17.64 & $7.17 \mathrm{E}-02$ & $1.40 \mathrm{E}+02$ & $4.44 \mathrm{E}-02$ & $6.05 \mathrm{E}+01$ & $8.43 \mathrm{E}+02$ & $288.3 \pm 7.6$ \\
\hline 10 & 20.47 & $7.90 \mathrm{E}-02$ & $1.37 \mathrm{E}+02$ & $4.78 \mathrm{E}-02$ & $6.36 \mathrm{E}+01$ & $8.04 \mathrm{E}+02$ & $291.8 \pm 7.1$ \\
\hline 11 & 17.06 & $6.59 \mathrm{E}-02$ & $1.30 \mathrm{E}+02$ & $5.16 \mathrm{E}-02$ & $5.68 \mathrm{E}+01$ & $8.61 \mathrm{E}+02$ & $272.1 \pm 6.8$ \\
\hline 12 & 21.3 & $8.06 \mathrm{E}-02$ & $1.45 \mathrm{E}+02$ & $5.66 \mathrm{E}-02$ & $6.05 \mathrm{E}+01$ & $7.50 \mathrm{E}+02$ & $277.5 \pm 7.8$ \\
\hline 13 & 16.03 & $6.72 \mathrm{E}-02$ & $1.43 \mathrm{E}+02$ & $3.67 \mathrm{E}-02$ & $5.67 \mathrm{E}+01$ & $8.44 \mathrm{E}+02$ & $277.2 \pm 7.2$ \\
\hline 14 & 12.53 & $5.93 \mathrm{E}-02$ & $1.40 \mathrm{E}+02$ & $4.33 \mathrm{E}-02$ & $5.54 \mathrm{E}+01$ & $9.35 \mathrm{E}+02$ & $281.2 \pm 6.9$ \\
\hline 15 & 13.66 & $5.89 \mathrm{E}-02$ & $1.32 \mathrm{E}+02$ & $6.50 \mathrm{E}-02$ & $5.47 \mathrm{E}+01$ & $9.28 \mathrm{E}+02$ & $273.1 \pm 6.5$ \\
\hline \multicolumn{8}{|c|}{$\mathrm{J}$-value $=0.00316449 \pm 0.00000357$} \\
\hline \multicolumn{8}{|c|}{ Mean Age $=276.1(\mathrm{STDEV}=12.0 \mathrm{Ma})$} \\
\hline
\end{tabular}

\begin{tabular}{rcccccccc}
\hline \multicolumn{7}{c}{ LJT2 Tremolite } \\
\hline 1 & 27.3 & $1.78 \mathrm{E}-01$ & $9.37 \mathrm{E}+01$ & $1.32 \mathrm{E}-01$ & $1.67 \mathrm{E}+02$ & $9.38 \mathrm{E}+02$ & $502.6 \pm 5.9$ \\
2 & 16.2 & $1.25 \mathrm{E}-01$ & $1.14 \mathrm{E}+02$ & $8.80 \mathrm{E}-02$ & $1.74 \mathrm{E}+02$ & $1.40 \mathrm{E}+03$ & $596.1 \pm 5.5$ \\
3 & 9.46 & $9.51 \mathrm{E}-02$ & $1.27 \mathrm{E}+02$ & $5.76 \mathrm{E}-02$ & $1.96 \mathrm{E}+02$ & $2.06 \mathrm{E}+03$ & $706.8 \pm 5.4$ \\
4 & 23.15 & $1.65 \mathrm{E}-01$ & $1.00 \mathrm{E}+02$ & $1.08 \mathrm{E}-01$ & $1.78 \mathrm{E}+02$ & $1.08 \mathrm{E}+03$ & $558.7 \pm 4.4$ \\
5 & 15.15 & $1.22 \mathrm{E}-01$ & $1.17 \mathrm{E}+02$ & $8.41 \mathrm{E}-02$ & $1.80 \mathrm{E}+02$ & $1.47 \mathrm{E}+03$ & $618.4 \pm 3.3$ \\
6 & 25.45 & $1.73 \mathrm{E}-01$ & $9.78 \mathrm{E}+01$ & $1.08 \mathrm{E}-01$ & $1.72 \mathrm{E}+02$ & $9.94 \mathrm{E}+02$ & $527.4 \pm 4.3$ \\
7 & 18.89 & $1.42 \mathrm{E}-01$ & $1.02 \mathrm{E}+02$ & $8.66 \mathrm{E}-02$ & $1.82 \mathrm{E}+02$ & $1.28 \mathrm{E}+03$ & $597.0 \pm 4.9$ \\
8 & 25.67 & $1.87 \mathrm{E}-01$ & $1.02 \mathrm{E}+02$ & $9.63 \mathrm{E}-02$ & $1.86 \mathrm{E}+02$ & $9.91 \mathrm{E}+02$ & $564.0 \pm 7.1$ \\
9 & 10.52 & $9.68 \mathrm{E}-02$ & $1.19 \mathrm{E}+02$ & $6.26 \mathrm{E}-02$ & $1.87 \mathrm{E}+02$ & $1.93 \mathrm{E}+03$ & $669.8 \pm 2.5$ \\
10 & 12.09 & $1.08 \mathrm{E}-01$ & $1.19 \mathrm{E}+02$ & $6.47 \mathrm{E}-02$ & $1.91 \mathrm{E}+02$ & $1.76 \mathrm{E}+03$ & $672.0 \pm 4.2$ \\
11 & 12.09 & $1.08 \mathrm{E}-01$ & $1.19 \mathrm{E}+02$ & $6.47 \mathrm{E}-02$ & $1.91 \mathrm{E}+02$ & $1.76 \mathrm{E}+03$ & $672.0 \pm 4.2$ \\
\hline
\end{tabular}


Table 1. (Continued)

\begin{tabular}{|c|c|c|c|c|c|c|c|}
\hline No. & Atm. $\%$ & ${ }^{36} \mathrm{Ar} /{ }^{39} \mathrm{Ar}$ & ${ }^{37} \mathrm{Ar} /{ }^{39} \mathrm{Ar}$ & ${ }^{38} \mathrm{Ar} /{ }^{39} \mathrm{Ar}$ & ${ }^{40} \mathrm{Ar} /{ }^{39} \mathrm{Ar}$ & ${ }^{40} \mathrm{Ar} /{ }^{36} \mathrm{Ar}$ & Date (Ma) \\
\hline \multicolumn{8}{|c|}{ LJT2 Tremolite } \\
\hline 12 & 33.85 & $2.25 \mathrm{E}-01$ & $8.87 \mathrm{E}+01$ & $1.14 \mathrm{E}-01$ & $1.77 \mathrm{E}+02$ & $7.86 \mathrm{E}+02$ & $484.5 \pm 4.8$ \\
\hline \multicolumn{8}{|c|}{$\mathrm{J}$-value $=0.00248087 \pm 0.00000405$} \\
\hline \multicolumn{8}{|c|}{ Mean Age $=597.5 \mathrm{Ma}(\mathrm{STDEV}=72.6 \mathrm{Ma})$} \\
\hline \multicolumn{8}{|c|}{ LJT35 Tremolite } \\
\hline 1 & 13.36 & $5.00 \mathrm{E}-01$ & $3.47 \mathrm{E}+02$ & $2.40 \mathrm{E}-01$ & $9.11 \mathrm{E}+02$ & $1.82 \mathrm{E}+03$ & $2267.4 \pm 21.9$ \\
\hline 2 & 6.32 & $2.60 \mathrm{E}-01$ & $2.42 \mathrm{E}+02$ & $2.29 \mathrm{E}-01$ & $9.29 \mathrm{E}+02$ & $3.57 \mathrm{E}+03$ & $2285 \pm 15.8$ \\
\hline 3 & 21.38 & $5.02 \mathrm{E}-01$ & $3.21 \mathrm{E}+02$ & $2.70 \mathrm{E}-01$ & $5.81 \mathrm{E}+02$ & $1.16 \mathrm{E}+03$ & $1597.9 \pm 13.6$ \\
\hline 4 & 16.43 & $3.43 \mathrm{E}-01$ & $1.86 \mathrm{E}+02$ & $1.72 \mathrm{E}-01$ & $5.32 \mathrm{E}+02$ & $1.55 \mathrm{E}+03$ & $1461.3 \pm 11.3$ \\
\hline 5 & 17.49 & $4.37 \mathrm{E}-01$ & $2.43 \mathrm{E}+02$ & $1.85 \mathrm{E}-01$ & $6.34 \mathrm{E}+02$ & $1.45 \mathrm{E}+03$ & $1677 \pm 17.5$ \\
\hline 6 & 10.23 & $3.12 \mathrm{E}-01$ & $1.63 \mathrm{E}+02$ & $1.85 \mathrm{E}-01$ & $7.80 \mathrm{E}+02$ & $2.50 \mathrm{E}+03$ & $1940.9 \pm 21.2$ \\
\hline 7 & 23.23 & $9.24 \mathrm{E}-01$ & $2.99 \mathrm{E}+02$ & $4.10 \mathrm{E}-01$ & $1.08 \mathrm{E}+03$ & $1.17 \mathrm{E}+03$ & $2279 \pm 26.4$ \\
\hline 8 & 12.72 & $3.72 \mathrm{E}-01$ & $2.71 \mathrm{E}+02$ & $2.16 \mathrm{E}-01$ & $7.03 \mathrm{E}+02$ & $1.89 \mathrm{E}+03$ & $1882.3 \pm 12.5$ \\
\hline 9 & 5.13 & $1.97 \mathrm{E}-01$ & $2.35 \mathrm{E}+02$ & $1.18 \mathrm{E}-01$ & $7.93 \mathrm{E}+02$ & $4.02 \mathrm{E}+03$ & $2093.6 \pm 18.5$ \\
\hline \multicolumn{8}{|c|}{$\mathrm{J}$-value $=0.00246837 \pm 0.00000405$} \\
\hline \multicolumn{8}{|c|}{ Mean Age $=1942.7 \mathrm{Ma}(\mathrm{STDEV}=312.9 \mathrm{Ma})$} \\
\hline
\end{tabular}

Note: J-value: Weighted mean of one fusion of irradiation standard LP-6 Biotite, having an Ar-Ar age of $128.4 \pm 0.2$ Ma (Renne et al. 1998). The date is obtained by using the following equations:

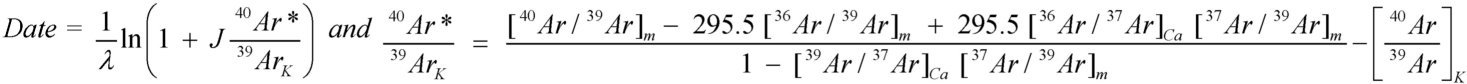

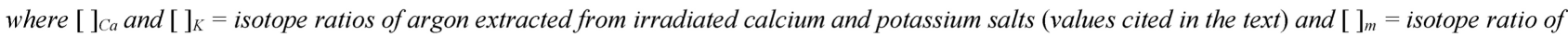
argon extracted from irradiated unknown.

Date $(\mathrm{Ma})=$ the date calculated using the following decay constants: $\lambda \varepsilon=0.581 \times 10^{-10} \mathrm{yr}^{-1} ; \lambda_{\beta}=4.962 \times 10^{-10} \mathrm{yr}^{-1} ; \lambda=5.543 \times 10^{-10} \mathrm{yr}^{-1} ;{ }^{40} \mathrm{~K} / \mathrm{K}=$ 0.01167 atom \% (Steiger and Jäger 1977).

The quoted error is one standard deviation and does not include the error in the standard error, or the error in the interference corrections.

Table 2. Summary of ${ }^{40} \mathrm{Ar} /{ }^{39} \mathrm{Ar}$ dating results conducted from samples.

\begin{tabular}{lccccccc}
\hline Location & sample & phase & Mean age (Ma) & Std $^{\mathbf{a}}$ & Intercept age (Ma) $^{\left.\mathbf{4 0}^{\mathbf{A}} \mathbf{A r}{ }^{\mathbf{3 6}} \mathbf{A r}\right)_{\mathbf{i}}}$ & MSWD $^{\mathbf{b}}$ \\
\hline MeiLing Jade & MLG1 & Tremolite & 110.9 & 7.5 & $117.0 \pm 3.2$ & $252 \pm 7$ & 3.587 \\
& MLW1 & Tremolite & 118.3 & 5.2 & $119.7 \pm 1.5$ & $285 \pm 2$ & 5.447 \\
\multirow{2}{*}{ QieMo Jade } & CM1 & Tremolite & 276.1 & 12 & $277.3 \pm 12.2$ & $288 \pm 37$ & 1.844 \\
LingJiaTan & LJT2 & Tremolite & 597.5 & 72.6 & & & \\
& LJT35 & Tremolite & 1942.7 & 312.9 & & & \\
\hline
\end{tabular}




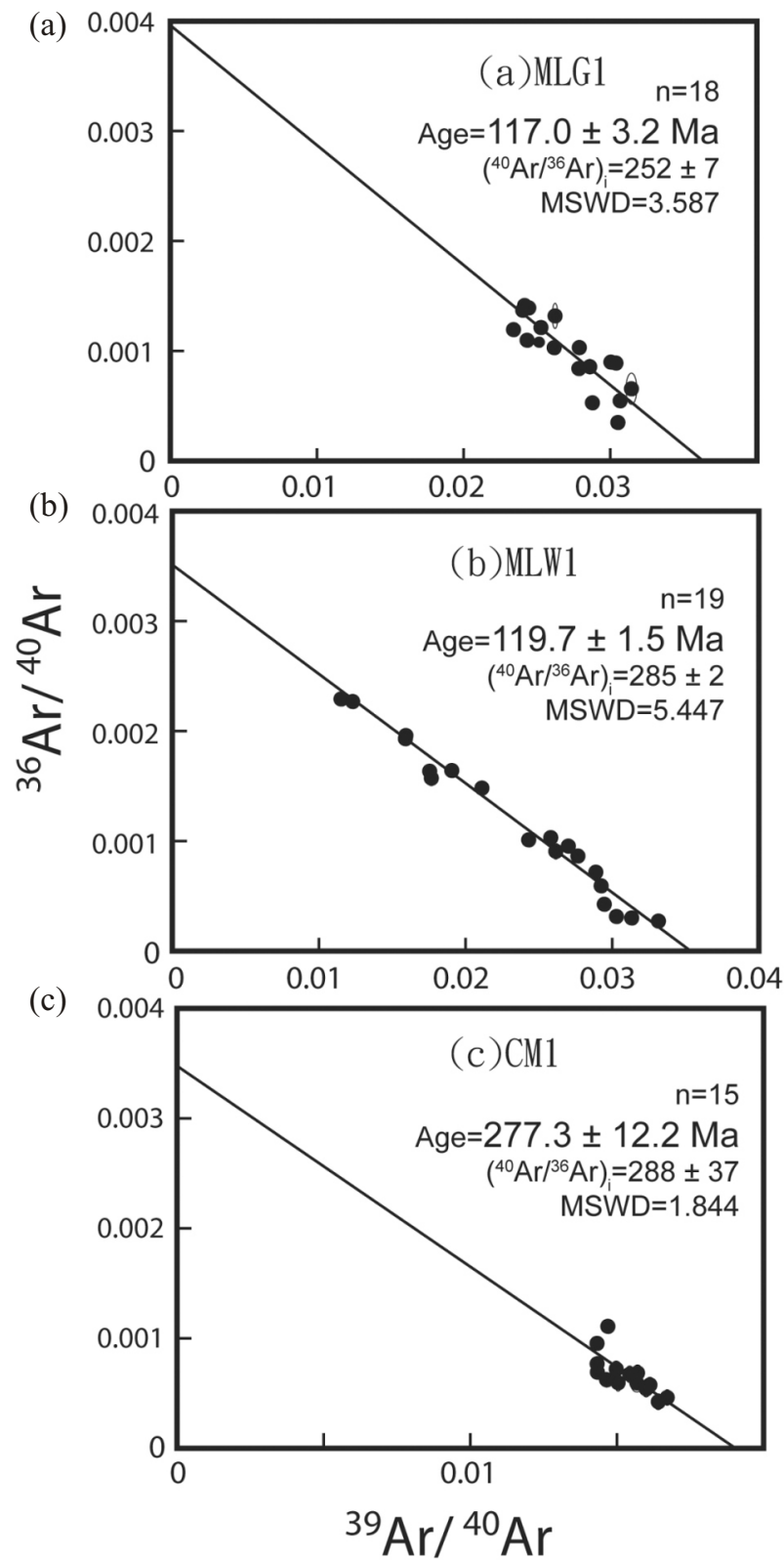

Fig. 4. Isotope correlation plots for tremolites: (a) MLG1 and (b) MLW1, from the ShiaoMeiLing jade mines and (c) CM1, from the QieMo jade mines.

crops showed normal isotope correlation plots. On the other hand, the archaic jade artifacts exhibit peculiar phenomena (Fig. 5).

As shown in Table 1, sample MLG1 from the ShiaoMeiLing jade mines, has ages in the range of 99.4 to $124.6 \mathrm{Ma}$, with a mean age of $110.9 \pm 7.5 \mathrm{Ma}$. In the isotope correlation diagram (Fig. 4a), regression of data yields an intercept date of $117.0 \pm 3.2 \mathrm{Ma}$, with an initial $\left({ }^{40} \mathrm{Ar} /{ }^{36} \mathrm{Ar}\right)_{\mathrm{i}}$ value around $252.4 \pm$ 7.3. Its MSWD (Mean Squared Weighted Deviation) is 3.587 , meaning that the sample has not been affected by any significant alteration effects. Raw jade sample MLW1 with white coloring showed an inter-

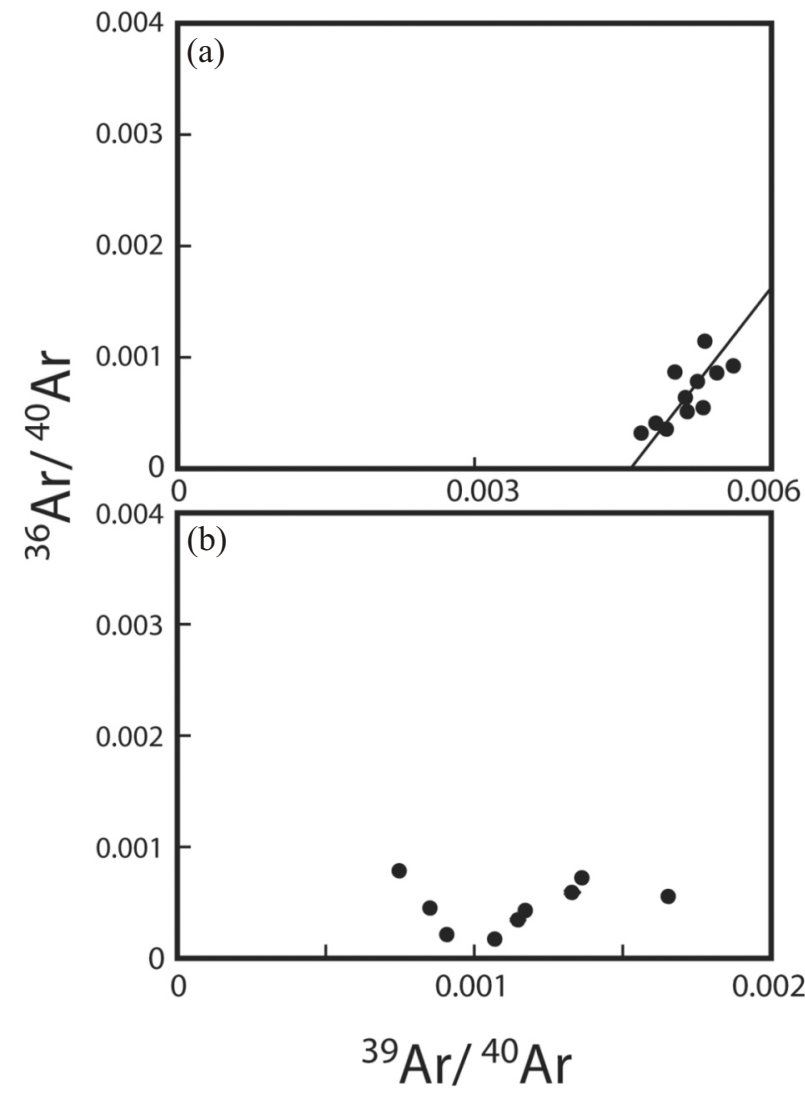

Fig. 5. Isotope correlation plots for the archaic jades: (a) LJT2 and (b) LJT35, from the LingJiaTan archaeological site.

cept date of $119.7 \pm 1.5 \mathrm{Ma}$. The initial $\left({ }^{40} \mathrm{Ar} /{ }^{36} \mathrm{Ar}\right)_{\mathrm{i}}$ value is $284.7 \pm 1.6$ and the MSWD value is 5.447 (Fig. 4b). All these obtained $\left({ }^{40} \mathrm{Ar} /{ }^{36} \mathrm{Ar}\right)_{\mathrm{i}}$ values are generally consistent with the atmospheric value (295.5). In addition, both samples, MLG1 and MLW1, reveal a similar isotope age which is in perfect agreement with the age of granitic intrusion in the same outcrop $(\sim 120 \mathrm{Ma})$ reported by Chou (2002). It is therefore believed that the formation age of the jade lode in mines at MeiLing Village should be around 117 to 119.7 Ma. It also confirms that the tremolite jades at the MeiLing Village were formed by contact metamorphism of the Yanshanian granite emplacement, as suggested by previous authors (Zhong 2000).

Sample CM1 collected from jade mines at QieMo County of XinJiang Province, showed isotope dates of 243.9 to $292.8 \mathrm{Ma}$ with mean age of $276.1 \pm 12.0 \mathrm{Ma}$. As seen in Fig. 4c, its isotope correlation plot shows a smooth linearity of the inverse isochron line with an intercept date of $277.3 \pm 12.2 \mathrm{Ma}$, an initial $\left({ }^{40} \mathrm{Ar} /{ }^{36} \mathrm{Ar}\right){ }_{\mathrm{i}}$ value of $287.6 \pm$ 36.7, and MSWD of 1.844. This also confirms that the HeTien Jades were formed in late Paleozoic, as suggested by Tang et al. (2002).

The archaic jade, LJT2, however showed a wide span of between 484.5 to $706.8 \mathrm{Ma}$ with an average age of 
$597.5 \pm 72.6$ Ma. Figure 5a shows an isotope correlation plot for sample LJT2, the distribution of data points demonstrates an opposite linear tendency compared to that of modern jade materials with an intercept date of 796.2 \pm 17.6 Ma and an unusual initial ${ }^{40} \mathrm{Ar} /{ }^{36} \mathrm{Ar}$ value of $-249.3 \pm$ 14.6. Similarly, the archaic jade sample LJT35 also shows aberrant isotope dates widely ranging from 1461.3 to 2285.0 Ma, with a meaningless mean age at $1942.7 \mathrm{Ma}$ (Table 1).

Aberrations in the argon testing data were not uncommon and frequently seen in argon dating experiments. More than 20 artifacts of archaic jade from various archaeological sites showed similar scattering results in the previous study (Chou 2002). One might consider that in addition to radiogenic decay and atmospheric contamination, there might be other argon sources for isotopes in the archaic jade artifacts. Considering the exposure the archaic jade artifacts have had, being buried for a long period of time compared to the raw jade materials, weathering effects might be an important factor and responsible for these abnormal dates. A previous study suggested that most archaic jades at LingJiaTan archaeological site were subjected to secondary alteration reactions mainly kaolinitization and calcification, showing chicken-white or pale-yellow colors, which often result in loss of potassium, gain or loss of calcium, and addition of water or chlorine in samples (Fong et al. 2005). Certainly, these reactions will also cause the disturbance of $\mathrm{K}-\mathrm{Ar}$ isotope systematics in mineral. Hence, more caution must be paid in dating archaic jades.

During argon dating, neutron irradiation not only generates ${ }^{39} \mathrm{Ar}_{\mathrm{k}}$, but also results in the conversion of calcium and chlorine into ${ }^{37} \mathrm{Ar}_{\mathrm{ca}}$ and ${ }^{38} \mathrm{Ar}_{\mathrm{cl}}$, (McDougall and Harrison 1999), respectively, if there is any addition of chlorine or loss of $\mathrm{K}$ and $\mathrm{Ca}$ in samples during alteration reactions. In terms of archaic jade artifacts, these decreases in both potassium and calcium content and simultaneous increase in chlorine content have been observed in previous reports and been attributed to weathering effects (Chou 2002; Fong et al. 2005). They should be proportionally reflected in the ratios of ${ }^{38} \mathrm{Ar}_{\mathrm{cl}}{ }^{39} \mathrm{Ar}_{\mathrm{k}}$ and ${ }^{37} \mathrm{Ar}_{\mathrm{ca}}{ }^{39} \mathrm{Ar}_{\mathrm{k}}$. Based on these considerations, the obtained data for archaic samples, LJT2 and LJT35, are replotted in the diagrams of ${ }^{38} \mathrm{Ar}_{\mathrm{cl}} /{ }^{39} \mathrm{Ar}_{\mathrm{k}}$ versus age and ${ }^{37} \mathrm{Ar}_{\mathrm{ca}}{ }^{39} \mathrm{Ar}_{\mathrm{k}}$ versus age. As shown in Fig. 6, the extrapolated asymptotic line reveals that $\mathrm{Ca} / \mathrm{K}$ ratio increased with age, but $\mathrm{Cl} / \mathrm{K}$ ratio decreased with age. The $\mathrm{Ca} / \mathrm{K}$ ratio in Fig. 6 spans a range of 313 to 460 , which is much higher than what is usually observed in tremolites from the ShiaoMeiLing jade mines (10.34 - 68.61) (Chou 2002). This evidently indicates that the studied samples have been subjected to a variable degree of alteration effects. With consideration given to the possible propagation of weathering effects, and the $\mathrm{Ca} / \mathrm{K}$ values of fresh tremolites, the original ages should be outlined by the gray area in Fig. 6. The intercept date extrapolated from this gray area suggests for sample LJT2 an age range between 40 to $120 \mathrm{Ma}$. These estimates for the age of the archaic jades from LingJiaTan archaeological site (LJT2) are generally in agreement with that of MLG1 and MLW1 tremolite samples from ShiaoMeiLing jade mines ( $120 \mathrm{Ma})$ (Table 2).

\section{CONCLUSION}

MeiLing jade and QieMo jade are comprised primarily of tremolite. Both show sporadic formations of chlorite and similar coloring, either opaque white or light green. Therefore, it is difficult to use the appearance or chemical composition of the jades to distinguish which of these is the original source of raw jade material in archaic jade artifacts found at the LingJiaTan archaeological site. On the other hand, if the formation age of raw jade materials is taken into account, the archaic jade artifacts reveal an isotopic age of less than 120 $\mathrm{Ma}$, which is similar to that of raw jade materials found at ShiaoMeiLing Village in JiangSu Province. Considering the large geographical distance between LingJiaTan and QieMo Counties, and the large difference in isotopic ages, $\sim 277.3$ Ma for QieMo jade and $\sim 120$ Ma for MeiLing jade, we suggest that artisans living at the LingJiaTan archaeological site in the Neolithic age had used jade sourced from the closer jade mines. In other words, there was no culture interaction, nor jade trade, between East China and XingJiang during the
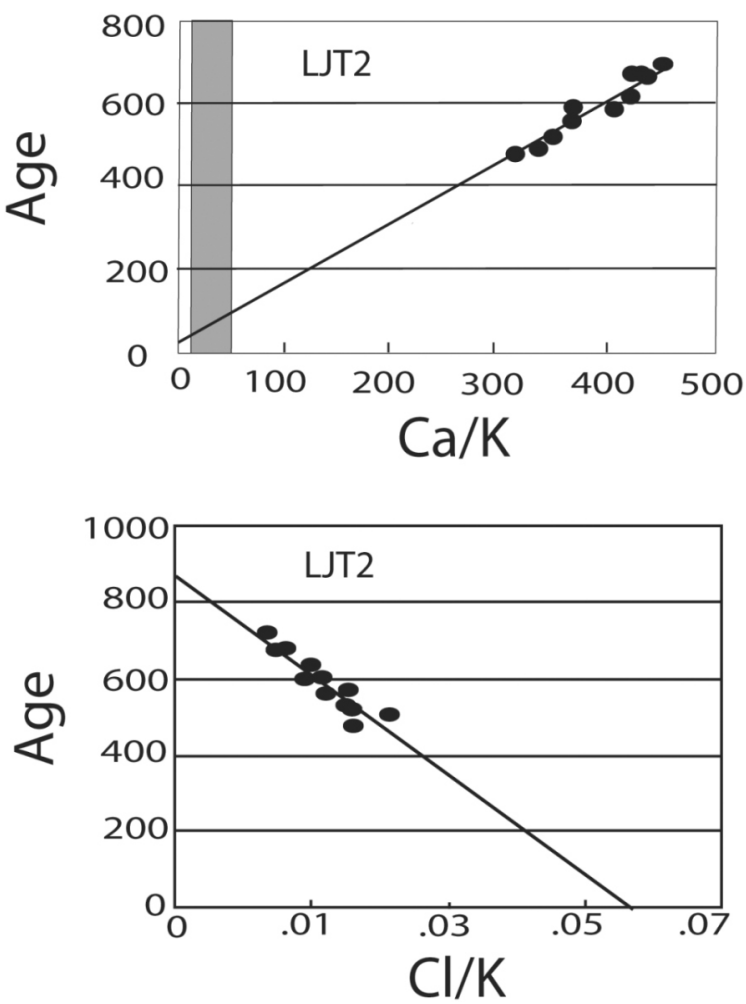

Fig. 6. ${ }^{37} \mathrm{Ar}_{\mathrm{ca}}{ }^{39} \mathrm{Ar}_{\mathrm{k}}$ and ${ }^{38} \mathrm{Ar}_{\mathrm{cl}}{ }^{39} \mathrm{Ar}_{\mathrm{k}}$ versus age plot for the archaic jade LJT2. 
Neolithic Period. This conclusion is contrary to the assumptions made in previous reports ( $\mathrm{Na} 1980$; Yang 1998).

Acknowledgements We are grateful to the Institute of Earth Sciences, Academia Sinica and the THOR Reactor, National Tsing Hua University, for their help in analysis. The help afforded by Yang, Boda, and Anhui Provincial Institute of Cultural Relics is also greatly appreciated.

\section{REFERENCES}

AnHui Provincial Institute of Cultural Relics, 2000: Jade Unearthed from LingJiaTan. Cultural Relics Publishing House, AnHui Province, 160 pp. (in Chinese)

Chou, S. J., 2002: ${ }^{40} \mathrm{Ar} /{ }^{39} \mathrm{Ar}$ ages of archaic jade and their implications to cultural exchange in China. Master Thesis, National Taiwan University, Taipei, Taiwan, 168 pp. (in Chinese)

Cui, W. Y., W. J. Wu, and Y. Liu, ,2002: Study on Liyang tremolite jade. Acta Petrol. Mineral., 21(Suppl.), 91-98. (in Chinese)

Fong, M., J. G. Zhang, R. Wang, and C. S. Wang, 2005: The analysis of impurity infiltration processes in ancient jade found at LingJiaTan. In: Yang, B. D., (Ed), The Proceedings of the $4^{\text {th }}$ Conference of Academic Study on Jade Culture, Forbidden City Press, 404-416. (in Chinese)

Kolesnik, Y. M., 1966: Nephrite of Siberia, American Geological Institute, Nauka Press, Siberian Division, Novo- sibirsk, $150 \mathrm{pp}$.

Lo, C. H., S. L. Chung, T. Y. Lee, and G. Y. Wu, 2002: Age of the Emeishan flood magmatism and relations to PermianTriassic boundary events. Earth Planet. Sci. Lett., 198, 449-458, doi: 10.1016/S0012-821X(02)00535-6. [Link]

McDougall, I. and T. M. Harrison, 1999: Geochronology and Thermochronology by the ${ }^{40} \mathrm{Ar} /{ }^{39} \mathrm{Ar}$ Method. Oxford Univ. Press, 269 pp.

Na, T. L., 1980: Chronological Ancient Chinese Articles Survey. Wenwen Publishing House, 47-97. (in Chinese)

Renne, P. R., C. C. Swisher, A. L. Deino, D. B. Karner, T. L. Owens, and D. J. DePaolo, 1998: Intercalibration of standards, absolute ages and uncertainties in ${ }^{40} \mathrm{Ar}{ }^{39} \mathrm{Ar}$ dating. Chem. Geol., 145, 117-152, doi: 10.1016/S0009-2541(97) 00159-9. [Link]

Tang, Y. L., D. Q. Liu, and, R. H. Zhou, 2002: Study on name, culture, quality and mineral deposit of Hetian jade. Acta Petrol. Mineral., 21(Suppl.), 13-21. (in Chinese)

Yang, B. D., 1998: Introduction to ancient Chinese jade articles. In: Yang, B. D., (Ed), History of Ancient Jade Articles, Forbidden City Press, 17-54. (in Chinese)

Zhong, H. B., 2000: Study on the geological characteristics and genesis of MeiLing Jade. J. Gems Gemol., 2, 39-44.

Zhong, H. B. and H. S. Zhang, 2002: Characteristics of Meiling jade from Jiangsu. Acta Petrol. Mineral., 21(Suppl.), 105-109. (in Chinese) 